\title{
A Robust Dynamic Inversion Technique for Asymptotic Tracking Control of an Aircraft
}

\author{
Ilker Tanyer, Enver Tatlicioglu, and Erkan Zergeroglu
}

\begin{abstract}
In this paper, a tracking controller is developed for an aircraft model subject to uncertainties in the dynamics and additive state-dependent nonlinear disturbance-like terms. In the design of the controller, dynamic inversion technique is utilized in conjuction with a robust term. Only the output of aircraft dynamics is utilized in the controller design and acceleration measurements are not required. Lyapunov based stability analysis is used to prove global asymptotic tracking.
\end{abstract}

\section{INTRODUCTION}

Dynamic inversion (DI) technique, which is a control design approach for nonlinear systems, was firstly developed for, and generally used in aerospace systems [1], [2]. The main idea of this technique is to transform the nonlinear system to a linear time invariant system by making change of variables and after using an appropriate control input, to drive the linear aircraft dynamics to a reference model [3], [4], [5], [6], [7], [8]. Guarino et al. utilized DI technique in servo control design and also compared it with traditional feedback controllers [3]. In [4], Oppenheimer and Doman applied the DI technique to stabilize an unstable, non-minimum phase hypersonic aircraft system. In [5], a DI based controller was proposed for Wiener systems. In [6], a DI based method was developed for finite time stability of a class of nonlinear systems where the input matrix was full rank. In [7], an autonomous flight control system was developed for a small scale unmanned helicopter based on approximate DI method. In [8], closed-loop stability of a six degree-of-freedom nonlinear air-to-air missile was ensured with a DI based controller. A DI based aircraft controller was developed for autonomous operation of a linear Yamaha RMAX helicopter in [9]. DI based controllers were also applied to experimental systems as in [10] and [11].

In the literature, DI technique is generally utilized when system dynamics is known. However, in some cases, and specifically for flight systems, exact dynamics is not available. When the system dynamics is subject to uncertainties (be it structured or unstructured), DI based algorithms can have difficulty in compensating for these uncertainties due to the increase in inversion error. Another reason for the increase of inversion error is the uncertainties in the input matrix. To avoid the increase in inversion error, uncertainties must be compensated by fusing the DI technique with adaptive and/or robust techniques. Some past research was

I. Tanyer and E. Tatlicioglu are with the Department of Electrical \& Electronics Engineering, Izmir Institute of Technology, Urla, Izmir, 35430 Turkey. ilkertanyereiyte.edu.tr

E. Zergeroglu is with the Department of Computer Engineering, Gebze Institute of Technology, Gebze, Kocaeli, 41400 Turkey. devoted to fusing DI technique with robust controllers. In [12], a robust DI method based on sliding mode control was proposed for tracking control of an unpowered flying vehicle. Yamasaki et al. proposed a robust DI controller for tracking control of an unmanned aerial vehicle (UAV) [13]. In [14], A stochastic robust nonlinear control approach fused with DI technique was applied to a highly nonlinear complicated aircraft model. In [15], a nonlinear dynamic inversion controller is combined with a PI controller to linearize the dynamics of UAVs. Some other past research fused adaptive control techniques with DI to compensate for linearly parameterizable uncertainties. For example, in [16], DI technique based null-space injection controller, and in [17], an adaptive DI based switching control methodology was proposed to compensate for structured uncertainties. In [18], DI was used in conjuction with a nonlinear model reference adaptive controller (MRAC) based on neural networks. Chen et al. proposed an adaptive dynamic inversion (ADI) based feedback linearization control for a flexible spacecraft [19]. To compensate for modeling errors and external disturbances, Wang and Stengel designed an ADI controller for a miniature UAV [20]. In [21], Calise and Rysdek proposed an ADI controller which was a combination of adaptive feedforward neural networks with feedback linearization. Lavretsky and Hovakimyan designed a direct MRAC augmented with a DI controller [22]. ADI based controllers, while compensating for structured uncertainties, mostly failed to address unstructured uncertainties. To compensate for both structured and unstructured uncertainties, neural networks were utilized in conjuction with ADI based controllers [23], [24], [25], [26] and [27]. However, in these works, while boundedness of the tracking error was ensured, asymptotic tracking was lost. Recently, Shin et al. developed a position tracking control system for a rotorcraft-based unmanned aerial vehicle (RUAV) by using robust integral of the signum of the error (RISE) feedback and neural network feedforward terms [28], [29]. Different from typical neural network based robust controllers, this method guaranteed semi-global asymptotic tracking. In [30], MacKunis et al. fused the robust controller in [31], [32] with DI technique to achieve asymptotic output tracking for aircraft systems with an uncertain input matrix and subject to additive unknown nonlinear disturbances. However, the signum of the time derivative of the output was utilized (i.e., acceleration information was required) in the design of the controller. Acceleration measurements are widely used in aircraft systems for system identification or control design. While acceleration measurements are available for some aircraft systems, utilizing these measurements 
in control design may not be preferred from control theory perspective. Additionally, although accelerometers may be seen as good and practical solutions in system identification and control applications, there are several reasons for not using them in some applications. Firstly, aside from onerousness in implementation, one needs to deal with sensor-related issues such as calibration and possible sensor failures. One way to avoid calibration requirements and sensor failures is, if possible, not to use them. For some cases, using them may be considered as redundant due to their costs. While the costs of sensors are decreasing rapidly, using them still adds to the cost of the overall system. Furthermore, aside from these, it should also be noted that using an additional sensor complicates the sensing system.

In this paper, model reference tracking control of an uncertain aircraft model subject to uncertainties is discussed. Specifically, the state and the input matrices are considered to be uncertain, and the dynamics is subject to an additive statedependent nonlinear disturbance-like terms. Furthermore, to remove the need for acceleration measurements, we consider that only the output of the aircraft being available for control development. In the design of the controller, the robust integral of the sign of the error component in [31], [32] is utilized. Since the input matrix of the aircraft system is considered to be uncertain, a matrix decomposition is utilized in the development of the error system. The control design is based on Lyapunov based design and analysis techniques, and global asymptotic stability of the tracking error is ensured.

\section{AIRCRAFT MODEL}

Following aircraft model is considered [4], [16], [33], [34]

$$
\dot{x}=A x+f(x, t)+B u, y=C x
$$

where $x(t) \in \mathbb{R}^{n}$ is the state vector, $A \in \mathbb{R}^{n \times n}$ is the state matrix, $B \in \mathbb{R}^{n \times m}$ is the input matrix, $y(t) \in \mathbb{R}^{m}$ is the output, $C \in \mathbb{R}^{m \times n}$ is the output matrix, $u(t) \in \mathbb{R}^{m}$ is the control input, and $f(x, t) \in \mathbb{R}^{n}$ is a state-dependent nonlinear disturbance-like term representing gravity, inertial coupling and nonlinear gust modeling. The above model is assumed to satisfy the following properties.

Assumption 1: The model in (1) is controllable.

Assumption 2: The state-dependent nonlinear disturbance-like term $f(x, t)$ is continuously differentiable and bounded up to its first order time derivative (i.e., $f(x, t) \in \mathcal{C}^{1}$ and $\left.f(x, t), \dot{f}(x, t) \in \mathcal{L}_{\infty}\right)$.

\section{Control Design}

The control design objective is to develop a robust control law that ensures that the output of the aircraft model $y(t)$ tracks the output of a reference model that will be designed subsequently, and additionally, all closed-loop signals are required to remain bounded. In the subsequent development, $C$ is assumed to be known, while $A, B$, and $f(x, t)$ are considered to be uncertain, thus, will not be utilized in the control design. The subsequent development is derived based on the assumption that only the output $y(t)$ is measurable.
The reference model is represented as

$$
\dot{x}_{m}=A_{m} x_{m}+B_{m} u_{m}, y_{m}=C x_{m}
$$

where $x_{m}(t) \in \mathbb{R}^{n}$ is the reference state vector, $A_{m} \in$ $\mathbb{R}^{n \times n}$ is the reference state matrix, $B_{m} \in \mathbb{R}^{n \times m}$ is the reference input matrix, $u_{m}(t) \in \mathbb{R}^{m}$ is the reference input, $y_{m}(t) \in \mathbb{R}^{m}$ is the reference output, and $C$ is the same output matrix in (1). The reference state matrix $A_{m}$ is chosen to be Hurwitz, and the reference input $u_{m}(t)$ and its time derivative are designed as bounded functions. Linear analysis tools can then be utilized along with these assumptions to prove that $x_{m}(t), \dot{x}_{m}(t), \ddot{x}_{m}(t)$ and thus, $y_{m}(t), \dot{y}_{m}(t)$, $\ddot{y}_{m}(t)$ are bounded functions.

To quantify the tracking control objective, an output tracking error, denoted by $e(t) \in \mathbb{R}^{m}$, is defined as

$$
e \triangleq y-y_{m}=C\left(x-x_{m}\right) .
$$

In the subsequent development, the error system will be designed based on a filtered tracking error, denoted by $r(t) \in$ $\mathbb{R}^{m}$, which is defined as

$$
r \triangleq \dot{e}+\Lambda e
$$

where $\Lambda \in \mathbb{R}^{m \times m}$ is a constant, positive definite, diagonal control gain matrix. It is noted that since only $y(t)$ is available then $\dot{e}(t)$ and thus $r(t)$ are not measurable, and cannot be utilized in the control design.

Assumption 3: Since the number of states is strictly greater than the number of outputs (i.e., $n>m$ ), there may be some states that can not be observed through the output. The subsequent control development and stability analysis rely on the assumption that the state vector can be partitioned as

$$
x=x_{o}+x_{u}
$$

where $x_{o}(t) \in \mathbb{R}^{n}$ contains the observable states through the output, and $x_{u}(t) \in \mathbb{R}^{n}$ contains the unobservable states. Furthermore, the unobservable states can be partitioned as

$$
x_{u}=x_{u \rho}+x_{u \xi}
$$

where $x_{u \rho}(t), x_{u \xi}(t) \in \mathbb{R}^{n}$ contain the unobservable states that can be bounded by a function of error signals and a constant, respectively. Mathematically speaking, following bounds are assumed

$$
\left\|x_{u \rho}(t)\right\| \leq c_{1}\|z\| \text { and }\left\|x_{u \xi}(t)\right\| \leq \xi_{x_{u}}
$$

where $c_{1}, \xi_{x_{u}} \in \mathbb{R}$ are known positive bounding constants and $z \triangleq\left[e^{T}, r^{T}\right]^{T} \in \mathbb{R}^{2 m}$ is the combined error signal. A similar upper bound can be obtained for the components of $\dot{x}_{u}(t)$ in the sense that

$$
\left\|\dot{x}_{u \rho}(t)\right\| \leq c_{2}\|z\| \text { and }\left\|\dot{x}_{u \xi}(t)\right\| \leq \xi_{\dot{x}_{u}}
$$

where $c_{2}, \xi_{\dot{x}_{u}} \in \mathbb{R}$ are known positive bounding constants.

Similar to (5), the reference state vector $x_{m}(t)$ can be partitioned as

$$
x_{m}=x_{m o}+x_{m u}
$$


where $x_{m o}(t) \in \mathbb{R}^{n}$ contains the entries of the reference state vector corresponding to the observable states of the state vector, and $x_{m u}(t) \in \mathbb{R}^{n}$ contains the rest of the entries of the reference state vector.

After substituting (1)-(3) into (4), following expression can be obtained

$$
r=C A x+\Omega u+C f-C A_{m} x_{m}-C B_{m} u_{m}+\Lambda e
$$

where $\Omega \triangleq C B \in \mathbb{R}^{m \times m}$ is an auxiliary constant matrix. Since $B$ is uncertain, then $\Omega$ is uncertain as well. Furthermore, we do not know whether or not $\Omega$ is symmetric and/or positive definite. Given these restrictions, we consider the SDU decomposition of $\Omega$ as [35]

$$
\Omega=S D U
$$

where $S \in \mathbb{R}^{m \times m}$ is a symmetric positive-definite matrix, $D \in \mathbb{R}^{m \times m}$ is a diagonal matrix with entries \pm 1 and $U \in$ $\mathbb{R}^{m \times m}$ is a unity upper triangular matrix. Details of the SDU decomposition can be found in [36].

Remark 1: We evaluated the SDU decomposition of $\Omega$ for different aircraft models in the literature. For all these models, we observed that the diagonal matrix $D$ was equal to identity matrix. However, for the sake of completeness, the subsequent controller will be designed to be applicable to any diagonal matrix $D$ without imposing any restrictions, as long as it is available for control design.

After utilizing (11), the time derivative of the filtered tracking error $r(t)$ can be written as

$\dot{r}=C A \dot{x}+S D U \dot{u}+C \dot{f}-C A_{m} \dot{x}_{m}-C B_{m} \dot{u}_{m}+\Lambda \dot{e}$.

After premultiplying (12) with $M \triangleq S^{-1} \in \mathbb{R}^{m \times m}$, following expression can be obtained

$$
M \dot{r}=M\left[C A \dot{x}+C \dot{f}-C A_{m} \dot{x}_{m}-C B_{m} \dot{u}_{m}+\Lambda \dot{e}\right]+D U \dot{u} .
$$

It is noted that, since $S$ is symmetric and positive-definite, then so is $M$. An auxiliary signal, denoted by $N(x, \dot{x}, t) \in$ $\mathbb{R}^{m}$ is defined as

$$
N \triangleq M\left[C A \dot{x}+C \dot{f}-C A_{m} \dot{x}_{m}-C B_{m} \dot{u}_{m}+\Lambda \dot{e}\right]+e
$$

which can be utilized to rewrite the expression in (13) as

$$
M \dot{r}=N-e+D U \dot{u} .
$$

The auxiliary signal $N$ can be partitioned as

$$
N=N_{d}+\tilde{N}
$$

where $N_{d}(t) \in \mathbb{R}^{m}$ contains functions that can be bounded by constants

$$
N_{d} \triangleq M C A \dot{x}_{u \xi}+M C \dot{f}-M C B_{m} \dot{u}_{m}+M C\left(A-A_{m}\right) \dot{x}_{m o}
$$

and $\tilde{N}(x, \dot{x}, e, \dot{e}) \in \mathbb{R}^{m}$ is an auxiliary error-like term defined as follows

$$
\begin{aligned}
\tilde{N} \triangleq & M C\left[A\left(\dot{x}_{o}-\dot{x}_{m o}\right)+A \dot{x}_{u \rho}-A_{m} \dot{x}_{m u}\right] \\
& +M \Lambda(r-\Lambda e)+e .
\end{aligned}
$$

The main idea behind partitioning $N$ as in (16)-(19) is to make use of the following facts.

Remark 2: From Assumptions 1 and 3, and the assumption on boundedness of the reference model signals, it can be shown that $N_{d}(t)$ is a bounded function of time in the sense that $\left\|N_{d}\right\| \leq \zeta_{N_{d}} \forall t$ where $\zeta_{N_{d}} \in \mathbb{R}$ is a positive bounding constant. Or alternatively, $\left|N_{d, i}\right| \leq \zeta_{N_{d, i}} \forall t$ with $\zeta_{N_{d, i}} \in \mathbb{R}$ being positive bounding constants.

Remark 3: The auxiliary error like term in (19) can be upper bounded as

$$
\|\tilde{N}\| \leq \rho\|z\|
$$

where $\rho \in \mathbb{R}$ is a positive bounding constant.

Based on the subsequent stability analysis, the control input is designed as

$$
u=-D K\left[e(t)-e(0)+\Lambda \int_{0}^{t} e(\tau) d \tau\right]-D \Pi
$$

where $\Pi(t) \in \mathbb{R}^{m}$ is an auxiliary filter signal updated according to ${ }^{1}$

$$
\dot{\Pi}(t)=\beta \operatorname{Sgn}(e(t)) \text { with } \Pi(0)=0_{m \times 1}
$$

where $\beta \in \mathbb{R}^{m \times m}$ is a constant, positive-definite, diagonal control gain matrix, $\operatorname{Sgn}(\cdot)$ denotes the vector signum function, and $K \in \mathbb{R}^{m \times m}$ is a constant, positive-definite, diagonal control gain matrix and defined as

$$
K=I_{m}+k_{g} I_{m}+\operatorname{diag}\left\{k_{d, 1}, k_{d, 2}, \ldots, k_{d, m-1}, 0\right\}
$$

with $k_{g}, k_{d, 1}, \ldots, k_{d, m-1} \in \mathbb{R}$ being positive gains. The time derivative of the control input in (20) is obtained as

$$
\dot{u}=-D K r-D \beta \operatorname{Sgn}(e)
$$

where (4) and (21) were utilized. After substituting (23) into (15), following closed-loop error system is obtained

$$
M \dot{r}=N_{d}+\tilde{N}-e-D U D \beta \operatorname{Sgn}(e)-D\left(U-I_{m}\right) D K r-K r
$$

where (16) was utilized.

Since $U$ is unity upper triangular then $U-I_{m}$ is strictly upper triangular, thus we can rewrite the $D\left(U-I_{m}\right) D K r$ term as

$$
D\left(U-I_{m}\right) D K r=\left[\Phi^{T}, 0\right]^{T}
$$

where the entries of $\Phi(r) \in \mathbb{R}^{(m-1) \times 1}$ are defined as

$$
\Phi_{i}=d_{i} \sum_{j=i+1}^{m} d_{j} k_{j} U_{i, j} r_{j} \text { for } i=1, \ldots,(m-1) .
$$

Since $d_{i}= \pm 1 \forall i=1, . ., m$, following upper bound can be obtained for the entries of $\Phi$

$$
\left|\Phi_{i}\right| \leq \sum_{j=i+1}^{m} k_{j} \zeta_{U_{i, j}}\left|r_{j}\right| \leq \zeta_{\Phi_{i}}\|z\|
$$

where $\zeta_{U_{i, j}}$ are positive bounding constants satisfying $\zeta_{U_{i, j}} \geq U_{i, j} \forall i, j$. It is important to highlight that $\zeta_{\Phi_{i}}$ depends on the control gains $k_{i+1}, \ldots, k_{m}$.

\footnotetext{
${ }^{1}$ Throughout the paper, $I_{n}$ and $0_{m \times r}$ will be used to represent an $n \times n$ standard identity matrix and an $m \times r$ zero matrix, respectively.
} 


\section{Stability ANALysis}

Theorem 1: The controller given in (20), (21) ensures global asymptotic tracking in the sense that

$$
\|e(t)\| \rightarrow 0 \text { as } t \rightarrow \infty
$$

provided that the control gain matrices $K$ and $\beta$ are selected by using the following procedure:

1) For $i=m, \beta_{m}$ is selected according to

$$
\beta_{m} \geq \zeta_{N_{d, m}}\left(1+\frac{\gamma_{2}}{\Lambda_{m}}\right)
$$

and from $i=m-1$ to $i=1, \beta_{i}$ are selected according to

$$
\beta_{i} \geq\left(\zeta_{N_{d, i}}+\sum_{j=i+1}^{m} \zeta_{\Psi_{j}} \beta_{j}\right)\left(1+\frac{\gamma_{2}}{\Lambda_{i}}\right)
$$

where $\gamma_{2} \in \mathbb{R}$ is some positive bounding constant and the subscript $i=1, \ldots, m$ denotes the $i$-th element of the vector or the diagonal matrix.

2) Control gain $k_{g}$ is chosen big enough to decrease the constant $\frac{\rho^{2}}{4 k_{g}}$.

3) Choose $k_{d, i}, i=1, \ldots,(m-1)$ to decrease the constant $\sum_{i=1}^{m-1} \frac{\zeta_{\Phi_{i}^{2}}}{4 k_{d, i}}$.

Proof: The proof of theorem has four subproofs. In the first part, boundedness of all the signals under the closedloop operation will be presented (see Appendix I). Secondly, a lemma and its proof (which utilizes the boundedness of the error signals) will be presented (see Appendix II). The proof of this lemma will provide an upper bound on the terms $\int_{0}^{t}\left|\dot{e}_{i}(\tau)\right| d \tau$, which will then be utilized in the next part of the proof. In the third part, the positiveness of an auxiliary integral term is demonstrated (see Appendix III). Finally, the asymptotic tracking result is proven (see Appendix IV).

\section{CONCLUSION}

A robust controller was designed for an aircraft model subject to uncertainties in the dynamics and additive statedependent nonlinear disturbance-like terms. In the design of the controller, a DI technique was used in conjuction with robust integral of the sign of the error terms to compensate for the uncertainties in the dynamic model. Lyapunov type stability analysis techniques were utilized to ensure global asymptotic tracking of the output of a reference model.

When compared with the similar studies in the literature, the key contribution of the proposed work is that only the output of the aircraft model was utilized in the control design and no acceleration measurements were required. Specifically, the closest work to ours is the work of MacKunis et al. in [30] where adaptive and robust controllers were designed for uncertain aircraft models subject to uncertainties in the dynamics and additive state-dependent nonlinear disturbance-like terms. In the design of the controllers, a DI technique was used in conjuction with robust integral of the sign of the error terms to obtain a similar result. However, in the design of the controllers in [30] the time derivative of the output was utilized, while in our work only the output information was utilized in the design of the controller.

\section{APPENDIX I \\ BOUNDEDNESS PROOF}

In this appendix, the boundedness of all the signals under the closed-loop operation will be demonstrated. Let $V_{1}(z) \in$ $\mathbb{R}$ be a Lyapunov function defined as

$$
V_{1} \triangleq \frac{1}{2} e^{T} e+\frac{1}{2} r^{T} M r
$$

which can be upper and lower bounded as

$$
\frac{1}{2} \min \left\{1, M_{\min }\right\}\|z\|^{2} \leq V_{1}(z) \leq \frac{1}{2} \max \left\{1, M_{\max }\right\}\|z\|^{2}
$$

where $M_{\min }$ and $M_{\max }$ denote minimum and maximum eigenvalues of $M$, respectively. Time derivative of the Lyapunov function can be written as

$$
\begin{aligned}
\dot{V}_{1} & =-e^{T} \Lambda e+r^{T} N_{d}+r^{T} \tilde{N}-r^{T} D U D \beta \operatorname{Sgn}(e) \\
& -r^{T}\left[\Phi^{T}, 0\right]^{T}-r^{T} r-k_{g} r^{T} r-\sum_{i=1}^{m-1} k_{d, i} r_{i}^{2} .
\end{aligned}
$$

After utilizing (27), following upper bound can be obtained

$$
r^{T}\left[\Phi^{T}, 0\right]^{T}=\sum_{i=1}^{m-1} r_{i} \Phi_{i} \leq \sum_{i=1}^{m-1} \zeta_{\Phi_{i}}\left|r_{i}\right|\|z\| .
$$

After substituting the upper bounds in Remarks 2 and 3, and utilizing (33), following expression can be obtained

$$
\begin{aligned}
\dot{V}_{1} & \leq-e^{T} \Lambda e-\|r\|^{2}+\zeta_{N_{d}}\|r\|+\zeta_{1}\|r\|+\rho\|r\|\|z\| \\
& -k_{g}\|r\|^{2}+\sum_{i=1}^{m-1} \zeta_{\Phi_{i}}\left|r_{i}\right|\|z\|-\sum_{i=1}^{m-1} k_{d, i} r_{i}^{2}
\end{aligned}
$$

where $r^{T} D U D \beta \operatorname{Sgn}(e) \leq \zeta_{1}\|r\|$ was utilized with $\zeta_{1} \in$ $\mathbb{R}$ being a positive bounding constant. After utilizing below manipulations

$$
\begin{aligned}
\zeta_{1}\|r\|+\zeta_{N_{d}}\|r\| & \leq \frac{1}{4 \delta}\|r\|^{2}+\delta\left(\zeta_{1}+\zeta_{N_{d}}\right)^{2} \\
\rho\|r\|\|z\|-k_{g}\|r\|^{2} & \leq \frac{\rho^{2}}{4 k_{g}}\|z\|^{2} \\
\zeta_{\Phi_{i}}\left|r_{i}\right|\|z\|-k_{d, i} r_{i}^{2} & \leq \frac{\zeta_{\Phi_{i}}^{2}}{4 k_{d, i}}\|z\|^{2}
\end{aligned}
$$

$\forall i=1, \ldots,(m-1)$, where $\delta \in \mathbb{R}$ is a positive damping constant, the right-hand side of (34) can be upper bounded as

$$
\begin{aligned}
\dot{V}_{1} & \leq-\left[\min \left\{\Lambda_{\min },\left(1-\frac{1}{4 \delta}\right)\right\}-\frac{\rho^{2}}{4 k_{g}}-\sum_{i=1}^{m-1} \frac{\zeta_{\Phi_{i}}^{2}}{4 k_{d, i}}\right]\|z\|^{2} \\
& +\delta\left(\zeta_{1}+\zeta_{N_{d}}\right)^{2}
\end{aligned}
$$

where $\Lambda_{\text {min }}$ denotes the minimum eigenvalue of $\Lambda$. Provided that the control gains $\Lambda, k_{g}, k_{d, 1}, \ldots, k_{d, m-1}$ are selected sufficiently high, the above expression can be rewritten as

$$
\dot{V}_{1} \leq-c_{1} V_{1}+c_{2}
$$


where $c_{1}$ and $c_{2}$ are some positive bounding constants. From (39), it can be concluded that $V_{1}(t) \in L_{\infty}$, and thus, $e(t)$, $r(t) \in L_{\infty}$. The definition of $r(t)$ in (4) can be utilized to prove that $\dot{e}(t) \in L_{\infty}$. By using (3) and its time derivative, along with the assumption that the reference model signals being bounded, it can be proven that $y(t), \dot{y}(t), x(t), \dot{x}(t) \in$ $\mathcal{L}_{\infty}$. The above boundedness statements and Assumption 2 can be utilized along with (1) to prove that $u(t) \in \mathcal{L}_{\infty}$. From (23), it is easy to see that $\dot{u}(t) \in \mathcal{L}_{\infty}$. After utilizing the above boundedness statements, Assumption 2, and the assumption that the reference model signals being bounded along with (12), it is clear that $\dot{r}(t) \in \mathcal{L}_{\infty}$. Standard signal chasing algorithms can be used to prove that all remaining signals are bounded.

\section{APPENDIX II}

\section{LEMMA 1 AND ITS PROOF}

Lemma 1: Provided that $e(t)$ and $\dot{e}(t)$ are bounded, the following expression for the upper bound of the integral of the absolute value of the $i$-th entry of $\dot{e}(t)$ can be obtained [41]

$$
\int_{t_{0}}^{t}\left|\dot{e}_{i}(\tau)\right| d \tau \leq \gamma_{1}+\gamma_{2} \int_{t_{0}}^{t}\left|e_{i}(\tau)\right| d \tau+\left|e_{i}\right|
$$

where $\gamma_{1}, \gamma_{2} \in \mathbb{R}$ are some positive bounding constants.

Proof: First, we note that if $e_{i}(t) \equiv 0$ on some interval, then $\dot{e}_{i}(t) \equiv 0$ on the same interval, and the inequality (40) yields this qualification. Therefore, without loss of generality, we assume that $e_{i}(t)$ is absolutely greater than zero on the interval of $\left[t_{0}, t\right]$. Let $T \in\left[t_{0}, t\right)$ be the last instant of time when $\dot{e}_{i}(t)$ changes sign. Then, on the interval $[T, t], \dot{e}_{i}(t)$ has a constant sign, hence

$$
\int_{T}^{t}\left|\dot{e}_{i}(\tau)\right| d \tau=\left|\int_{T}^{t} \dot{e}_{i}(\tau) d \tau\right|=\left|e_{i}(t)-e_{i}(T)\right| .
$$

From the boundedness of $\dot{e}_{i}(t)$, it follows that there exist a constant $\gamma>0$ such that $\left|\dot{e}_{i}(t)\right| \leq \gamma$, therefore

$$
\int_{t_{0}}^{T}\left|\dot{e}_{i}(\tau)\right| d \tau \leq \gamma\left(T-t_{0}\right)
$$

On the other hand, by applying the Mean Value Theorem [42], we can obtain the following expression

$$
\int_{t_{0}}^{T}\left|e_{i}(\tau)\right| d \tau=\left(T-t_{0}\right) e_{i *}
$$

where $e_{i *}$ is some intermediate value of $\left|e_{i}(t)\right|$ on the interval $\left[t_{0}, T\right]$. By assumption, $e_{i *}$ is bounded away from zero. Therefore, from (42) and (43), we can conclude as

$$
\int_{t_{0}}^{T}\left|\dot{e}_{i}(\tau)\right| d \tau \leq \gamma_{2} \int_{t_{0}}^{T}\left|e_{i}(\tau)\right| d \tau
$$

where $\gamma_{2} \triangleq \frac{\gamma}{e_{i *}}$. Combining the relationships in (41) and (44), we can write

$$
\int_{t_{0}}^{t}\left|\dot{e}_{i}(\tau)\right| d \tau \leq\left|e_{i}(t)\right|+\gamma_{2} \int_{t_{0}}^{t}\left|e_{i}(\tau)\right| d \tau+\left|e_{i}(T)\right|
$$

which yields in (40) with $\gamma_{1} \triangleq \sup \left|e_{i}(T)\right|$.

\section{APPENDIX III \\ LEMMA 2 AND ITS PROOF}

Lemma 2: Let the auxiliary function $L(t) \in \mathbb{R}$ be defined as

$$
L \triangleq r^{T}\left(N_{d}-D U D \beta \operatorname{Sgn}(e)\right) .
$$

If the entries of $\beta$ are selected to satisfy the conditions in (29) and (30), then it can be concluded that $P(t) \in \mathbb{R}$ defined as

$$
P \triangleq \zeta_{b}-\int_{0}^{t} L(\tau) d \tau
$$

is nonnegative where $\zeta_{b} \in \mathbb{R}$ is a positive bounding constant.

Proof: The proof can be found in [43].

\section{APPENDIX IV \\ ASYMPTOTIC STABILITY PROOF}

In this appendix, the asymptotic stability of the output tracking error is presented.

Let $V_{2}(w) \in \mathbb{R}$ be a Lyapunov function defined as

$$
V_{2} \triangleq V_{1}+P
$$

where $w(t) \triangleq\left[\begin{array}{lll}e^{T} & r^{T} & \sqrt{P}\end{array}\right]^{T} \in \mathbb{R}^{(2 m+1) \times 1}$. It should be noted that, the non-negativeness of $P(t)$, which is essential to prove that $V_{2}(w)$ is a valid Lyapunov function, was proven in Appendix III. The Lyapunov function in (48) can be upper and lower bounded as follows

$$
\frac{1}{2} \min \left\{1, M_{\min }\right\}\|w\|^{2} \leq V_{2}(w) \leq \max \left\{\frac{1}{2} M_{\max }, 1\right\}\|w\|^{2} .
$$

Taking the time derivative of the Lyapunov function in (48), substituting (32) and time derivative of (47), and after some straightforward manipulations, we obtain

$$
\begin{aligned}
\dot{V}_{2} & =-e^{T} \Lambda e+r^{T} \tilde{N}-r^{T}\left[\Phi^{T}, 0\right]^{T}-r^{T} r-k_{g} r^{T} r \\
& -\sum_{i=1}^{m-1} k_{d, i} r_{i}^{2} .
\end{aligned}
$$

After utilizing (36) and (37), the right-hand side of (49) can be upper bounded as

$$
\dot{V}_{2} \leq-\left[\min \left\{\lambda_{\min }(\Lambda), 1\right\}-\frac{\rho^{2}}{4 k_{g}}-\sum_{i=1}^{m-1} \frac{\zeta_{\Phi_{i}}^{2}}{4 k_{d, i}}\right]\|z\|^{2} .
$$

Provided that the control gains $\Lambda, k_{g}, k_{d, 1}, \ldots, k_{d, m-1}$ are selected sufficiently high, the below expression can be obtained for the derivative of the Lyapunov function

$$
\dot{V}_{2} \leq-c_{3}\|z\|^{2}
$$

where $c_{3}$ is some positive bounding constant. From (48) and (51), it is clear that $V_{2}(w)$ is nonincreasing and bounded. After integrating (51), it can be concluded that $z(t) \in \mathcal{L}_{2}$. Since $z(t) \in \mathcal{L}_{\infty} \cap \mathcal{L}_{2}$ and $\dot{z}(t) \in \mathcal{L}_{\infty}$, from Barbalat's Lemma [42], $\|z(t)\| \rightarrow 0$ as $t \rightarrow \infty$, thus meeting the control objective. Since no restrictions with respect to the initial conditions of the error signals were imposed on the control gains, the result is global. 


\section{REFERENCES}

[1] F. Lewis and B. L. Stevens, "Aircraft Control \& Simulation," New York, NY, USA: John Wiley \& Sons, 2003.

[2] D. Enns, D. Bugajski, R. Hendrick, and G. Stein, ”Dynamic inversion: An evolving methodology for flight control design," Int. J. of Cont., vol. 59, no. 1, pp. 71-91, 1994.

[3] C. Guarino, L. Bianco, and A. Piazzi, "A servo control system design using dynamic inversion," Cont. Eng. Pra., vol. 10, pp. 847-855, 2002.

[4] M. W. Oppenheimer and D. B. Doman, "Control of an unstable, nonminimum phase hypersonic vehicle model," IEEE Aerosp. Conf., Big Sky, MT, USA, 2006, pp. 1-7.

[5] Z. Szabo, P. Gaspar, and J. Bokor, "Tracking design for Wiener systems based on dynamic inversion," Int. Conf. on Cont. Appl., Munich, Germany, 2006, pp. 1386-1391.

[6] S. Onori, P. Dorato, S. Galeani, and C. Abdallah, "Finite time stability design via feedback linearization," IEEE Conf. on Decision \& Cont., Seville, Spain, 2005, pp. 4915-4920.

[7] Z. Zhang, F. Hu, and J. Li, "Autonomous flight control system designed for small-scale helicopter based on approximate dynamic inversion," Int. Conf. on Adv. Comp. Cont., 2011, pp. 185-191.

[8] C. J. Schumacher and P. P. Khargonekar, "Stability Analysis of a Missile Control System with a Dynamic Inversion Controller," J. of Guidance, Cont. \& Dynamics, vol. 21, no. 3, pp. 508-515, 1998.

[9] D. Enns and T. Keviczky, "Dynamic inversion based flight control for autonomous RMAX helicopter," American Cont. Conf., Minneapolis, MN, USA, 2006, pp. 3916-3923.

[10] C. Guowei, A. K. Cai, B. M. Chen, and T. H. Lee, "Construction, modeling and control of a mini autonomous UAV helicopter," IEE Int. Conf. Automation \& Logistics, 2008, pp. 449-454.

[11] K. Peng, M. Dong, B. M. Chen, G. Cai, K. Y. Lum, and T. H. Lee, "Design and implementation of a fully autonomous flight control system for a UAV helicopter," Chinese Cont. Conf., 2007, pp.662-667.

[12] Z. Liu, F. Zhou, and J. Zhou, "Flight control of unpowered flying vehicle based on robust dynamic inversion," Chinese Cont. Conf., Heilongjiang, China, 2006, pp. 693-698.

[13] T. Yamasaki, H. Sakaida, K. Enomoto, H. Takano, and Y. Baba, "Robust trajectory-tracking method for UAV guidance using proportional navigation," Int. Conf. on Cont. Autom. Sys., 2007, pp. 1404-1409.

[14] Q. Wang and R. F. Stengel, "Robust nonlinear flight control of a high performance aircraft," IEEE Tr. Cont. Sys. Tech., vol. 13, no. 1, pp. 15-26, 2005.

[15] Z. Xie, Y. Xia, and M. Fu, "Robust trajectory-tracking method for UAV using nonlinear dynamic inversion," IEEE Int. Conf. Cyber. \& Intel. Sys., 2011, pp. 93-98.

[16] A. D. Ngo and D. B. Doman, "Dynamic inversion-based adaptive/reconfigurable control of the X-33 on ascent," IEEE Aerosp. Conf., Big Sky, MT, USA, 2006, pp. 2683-2697.

[17] M. D. Tandale and J. Valasek, "Adaptive dynamic inversion control of a linear scalar plant with constrained control inputs," American Cont. Conf., Portland, OR, USA, 2005, pp. 2064-2069.

[18] X. J. Liu, F. Lara-Rosano, and C. W. Chan, "Model-reference adaptive control based on neurofuzzy networks," IEEE Tr. Sys. Man Cyber. C, Appl. Rev., vol. 34, no. 3, pp. 302-309, 2004.

[19] J. Chen, D. Li, X. Jiang, and X. Sun, "Adaptive feedback linearization control of a flexible spacecraft," Conf. Intell. Sys. Design Appl., Jinan, China, 2006, pp. 225-230.

[20] Q. Wang and R. F. Stengel, "A dynamic inversion controller design for miniature Unmanned Aerial Vehicles," Consumer Elec., Comm. \& Networks, pp. 1916-1921, 2011.

[21] A. Calise and R. Rysdyk, "Nonlinear adaptive flight control using neural networks," IEEE Cont. Sys. Mag., vol. 18, no. 6, pp. 14-25, 1998.

[22] E. Lavretsky and N. Hovakimyan, "Adaptive compensation of control dependent modeling uncertainties using time-scale separation," IEEE Conf. on Decision \& Cont., Seville, Spain, 2005, pp. 2230-2235.

[23] J. Leitner, A. Calise, and J. V. R. Prasad, "Analysis of adaptive neural networks for helicopter flight controls," J. Guidance Cont. Dynamics, vol. 20, no. 5, pp. 972-979, 1997.

[24] Y. Shin, "Neural network based adaptive control for nonlinear dynamic regimes," PhD diss., Georgia Inst. Tech., Atlanta, GA, USA, 2005.

[25] E. N. Johnson and A. J. Calise, "Pseudo-control hedging: A new method for adaptive control," Workshop Adv. Guid. Cont. Tech., Redstone Arsenal, AL, USA, 2000
[26] C. Schumacher and J. D. Johnson, "PI control of a tailless fighter aircraft with dynamic inversion and neural networks," American Cont. Conf., 1999, pp. 4173-4177.

[27] R. Rysdyk, F. Nardi, and A. J. Calise, "Robust adaptive nonlinear flight control applications using neural networks," American Cont. Conf., 1999 , pp. 2595-2599.

[28] J. Shin, H. J. Kim, K. Youdan, and W. E. Dixon, "Asymptotic attitude tracking of the rotorcraft-based UAV via RISE feedback and NN feedforward," IEEE Conf. Decision \& Cont., 2010, pp. 3694-3699.

[29] J. Shin, H. J. Kim, K. Youdan, and W. E. Dixon, "Autonomous Flight of the Rotorcraft-Based UAV Using RISE Feedback and NN Feedforward Terms," IEEE Tr. Cont. Sys. Tech., vol. 20, no. 5, pp. 1392-1399, 2012.

[30] W. MacKunis, P. M. Patre, M. K. Kaizer, and W. E. Dixon, "Asymptotic Tracking for Aircraft via Robust and Adaptive Dynamic Inversion Methods," IEEE Tr. Cont. Sys. Tech., vol. 18, no. 6, pp. 1448-1456, 2010.

[31] B. Xian, D. M. Dawson, M. S. de Queiroz, and J. Chen, "A continuous asymptotic tracking control strategy for uncertain nonlinear systems," IEEE Tr. Autom. Cont., vol. 49, no. 7, pp. 1206-1211, 2004.

[32] P. M. Patre, W. MacKunis, C. Makkar, and W. E. Dixon, "Asymptotic tracking for systems with structured and unstructured uncertainties," IEEE Conf. on Decision \& Cont., 2006, pp. 441-446.

[33] L. Duan, W. Lu, F. Mora-Camino, and T. Miquel, "Flight-path tracking control of a transportation aircraft: Comparison of two nonlinear design approaches," Digit. Avi. Sys. Conf., Portland, OR, USA, 2006, pp. 1-9.

[34] I. Szaszi, B. Kulcsar, G. J. Balas, and J. Bokor, 'Design of FDI filter for an aircraft control system," American Cont. Conf., 2002, pp. 42324237.

[35] R. R. Costa, L. Hsu, A. K. Imai, and P. Kokotovic, "Lyapunov-based adaptive control of MIMO systems," Automatica, vol. 39, no. 7, pp. $1251-1257,2003$

[36] G. Tao, "Adaptive Control Design and Analysis," New York, NY, USA: John Wiley \& Sons, 2003

[37] W. Mackunis, "Nonlinear Control for Systems Containing Input Uncertainty via a Lyapunov-Based Approach," Ph.D. dissertation, University of Florida, Gainesville, FL, USA, 2009.

[38] W. MacKunis, M. K. Kaiser, P. M. Patre, and W. E. Dixon, "Asymptotic tracking for aircraft via an uncertain dynamic inversion method," American Cont. Conf., Seattle, WA, USA, 2008, pp. 3482-3487.

[39] W. MacKunis, M. K. Kaiser, P. M. Patre, and W. E. Dixon, "Adaptive dynamic inversion for asymptotic tracking of an aircraft reference model," AIAA Guid. Nav. Cont. Conf., Honolulu, HI, USA, 2008, AIAA-2008-6792.

[40] Dep. of Transportation, Airworthiness Standards: Transport category airplanes, in Federal Aviation Regulations - Part 25, DC, USA, 1996.

[41] V. Stepanyan and A. Kurdila, "Asymptotic tracking of uncertain systems with continuous control using adaptive bounding," IEEE Tr. Neural Networks, vol. 20, no. 8, pp. 1320-1329, 2009.

[42] H. K. Khalil, "Nonlinear Systems," New York, NY, USA: Prentice Hall, 2002.

[43] B. Bidikli, E. Tatlicioglu, E. Zergeroglu, and A. Bayrak, "An Asymptotically Stable Continuous Robust Controller for a Class of Uncertain MIMO Nonlinear Systems," ArXiv e-prints:1301.5483, Jan. 2013. 